\title{
High Gain Dual-Polarized Non-uniform Spacing Stacked Patch Yagi-Uda Type Antenna
}

\author{
Muhammad Nazrul Islam*†, Markus Berg*, and Erkki T. Salonen* \\ ${ }^{*}$ Centre for Wireless Communication at the University of Oulu, Finland ${ }^{\dagger}$ Esju Oy, Konekuja 8, 90620 Oulu, \\ Finland
}

\begin{abstract}
This article describes a unique dual polarized antenna using stacked patches to increase gain similar as dipole array by Yagi-Uda. The antenna structure is very simple and useful for many wireless application where high gain is essential from single RF feed. Increasing gain by optimizing Yagi-Uda antenna director spacing and length are well studied topics where tradeoff is made between bandwidth and maximum gain. The key design uniqueness of the presented antenna is dual polarized feed patch antenna with higher gain and wider bandwidth. A detail step by step optimization technique for a $7 \mathrm{GHz}$ high gain stack patch antenna with $650 \mathrm{MHz}$ bandwidth is described. Measured realized maximum gain is over $12 \mathrm{dBi}$ with 4 directors. Simulation and measured results are compared. Simple stacking and feed method of the proposed high gain antenna will give easy manufacturing capability of this antenna and could be good candidate for millimeter and sub-millimeter wave RF system.
\end{abstract}

Index Terms-High gain, Dual polarized, Yagi-Uda antenna, Stack patch

\section{INTRODUCTION}

Patch antenna is a very useful directive planar antenna element used in wireless communication. Typical gain of a single patch antenna is 6-7 $\mathrm{dBi}$. By using larger ground plane or forming the ground plane the typical gain can be increased to around 8-9 dBi. Further increase of gain from single feed is challenging without the array form. Gain increase by planar phase array is a very common practice in case where high gain and beam steering is required. In phase array design planar transmission feeding network has certain loss contribution. The bigger the array the bigger the loss.

Massive MIMO implementation require many antennas elements and this number is smaller if individual antenna element has higher gain. High gain from a single feed antenna reduces the number of RF chains in massive MIMO application. High gain antenna element reduces the power consumption of the transmitter and receiver. Broad band high gain simple antenna structure solution is highly desirable for current wireless technology evolution.

Yagi-Uda antenna is a very popular choice to increase gain from one RF feed point. There are many Yagi-Uda research papers described gain increase optimization [1]-[4]. Analysis of Yagi-Uda antenna is described in [1], and it shows how a small change in the director length has effect of maximum gain. Using genetic algorithm to optimize the gain and input impedance was presented in [2]. In this optimization technique a wider bandwidth was not a optimization goal. Reported optimized gain with 6 elements was $12.58 \mathrm{dBi}$ and no data about impedance matched bandwidth reported in this article. Spacing optimization of antenna elements, directors and reflector to achieve higher gain and less side lobes is studied in [3]. Later with this optimized spacing array a element length optimization to achieve higher gain is studied. Both spacing and length optimized six element Yagi-Uda antenna [4] directivity is $15.5 \mathrm{dBi}$. Proposed optimized design didn't provide supported bandwidth and assume it is narrow band solution. Broad band one planar Quasi-Yagi antenna is reported in the literature [5]. Reported antenna has $48 \%(V S W R<2)$ bandwidth and has 3-5 dB gain. Another high gain design has $5.7 \mathrm{~dB}$ gain by reducing bandwidth to $11 \%$. A compact three-elements Yagi-Uda antenna is reported in [6]. In this reported design the drive element is a folded monopole. Directors and reflector were bowtie monopole to increased bandwidth. Reported 7\% bandwidth design has gain of 9.9-9.5 $\mathrm{dBi}$. A wide bandwidth (78.4\%) double-dipole quasi antenna is presented in [7]. A stable gain of 6.4-7.4 $\mathrm{dBi}$ for $76 \%$ bandwidth is reported there and front-to-back ratio is only $10 \mathrm{~dB}$. Optimization of bandwidth and gain by a vertical parasitic patch are studied in [8], [9]. Reference [8] shows bandwidth optimization by parasitic patch shapes and in [9] experimental results show 2nd parasitic distance $0.3 \lambda$ to increase the gain from single patch antenna similar like Yagi-Uda antenna. Microstrip-based Yagi-Uda or Quasi-Yagi antenna structures have been reported in [10], [11]. In [10], the reported antenna gain increase is done by using patch as reflector and director with driven patch element. By using active patch and parasitic patches (reflector and director elements) on the same horizontal plane, the main beam of the array has been tilted by the effect of mutual coupling instead of increasing the gain of the drive patch at the end fire direction. A single feed microstrip yagi antenna proposed in [11] provided simulation results for $\mathrm{mm}$-wave antenna and reported scaled version measured prototype of $5.2 \mathrm{GHz}$ antenna has $10.7 \mathrm{dBi}$ gain with $10 \%(S 11<-10 \mathrm{~dB})$ bandwidth. A stacked annular patch for Ku-band satellite communication is reported in [12]. Reported antenna with $2.2 \lambda$ ground plane $(56 \mathrm{~mm}$, center frequency $11.95 \mathrm{GHz}$ ) has $4 \%-10 \mathrm{~dB}$ reflection bandwidth and max gain around 12 $\mathrm{dB}$ at $12 \mathrm{GHz}$. A detail analysis of capacitive L-Probe feed patch antenna gain and pattern optimization on finite ground 
plane is studied in [13]. Article [14] reported a wide band single polarized magneto-electric dipole on a ground plane and achieved $95.2 \%$ bandwidth $(S W R \leq 2)$ with $7.9 \pm 0.9$ $\mathrm{dBi}$ gain. Optimization gain and operating bandwidth by vertically stacked multi layered slot patch antenna with single polarization is reported in [15]. Measured results [15] show peak gain $12.2 \mathrm{~dB}$ with $27.8 \%$ bandwidth $(S 11<-10 \mathrm{~dB})$ and gain drop around $3 \mathrm{~dB}$ from peak at the edge of the operating band width. A dual polarized Yagi type vertically stacked multilayered capacitive coupled patch antenna with $4 \%$ bandwidth is reported in [16]. Measured max gain at $5.8 \mathrm{GHz}$ with 4 directors is $9.76 \mathrm{~dB}$ but gain over operating frequencies is not reported.

None of these articles provide an antenna solution for dual polarized feed antenna with more than $12.5 \mathrm{dBi}$ realized gain and more than $9.5 \%$ band width.

In this paper a detail design procedure and optimization technique for a multilayer direct-feed dual polarized Yagi-uda type patch antenna array is described. Optimization is done to achieve better gain, larger bandwidth from both ports. The antenna is simulated by using CST microwave studio.

\section{ANTENNA STRUCTURE}

\section{A. Driven patch and Feed}

Proposed driven patch structure is shown in Fig. 1. Driven patch is feed by a $0.8 \mathrm{~mm}$ vertically placed cylindrical pin from bottom ground plane with CPW transmission line. 0.508 $\mathrm{mm}$ thick Rogers TR5880 with $35 \mu \mathrm{m}$ copper thickness is used as antenna PCB substrate. Detailed dimensions are given in Fig. 1. Drive patch extra extension above $14 \mathrm{~mm}$ square come from optimization for impedance matching and isolation improvement between two ports. Different shapes of drive patch (circular, elliptical), slots on drive patch and tapered drive patches are tried out for further optimization bandwidth. Because of no significance improvement noticed in comparison to this simple patch those are not reported here. However, after prototype tests, simulated isolation improvements are studied and results are presented in Section IV.

\section{B. Ground plane size}

Ground plane size has strong effect on patch antenna gain enhancement and for stacked patches it has more significant role because of bigger antenna volume. An analysis of ground plane size for multi stacked patch is done in [16]. In the proposed antenna, finding the ground plane size is done by simulation for the best maximum gain achieved over the broader bandwidth.

\section{Vertically stacked Yagi-Uda type patch antenna array}

To increase the gain from one feed point four stacked directors are placed vertically at the top of the drive patch. All directors have the same substrate material and the thickness is the same as the drive patch. Fig. 2 describes proposed antenna array simulation model in detail. Cylindrical antenna feed pin is connected to the PCB CPW transmission feed line and the
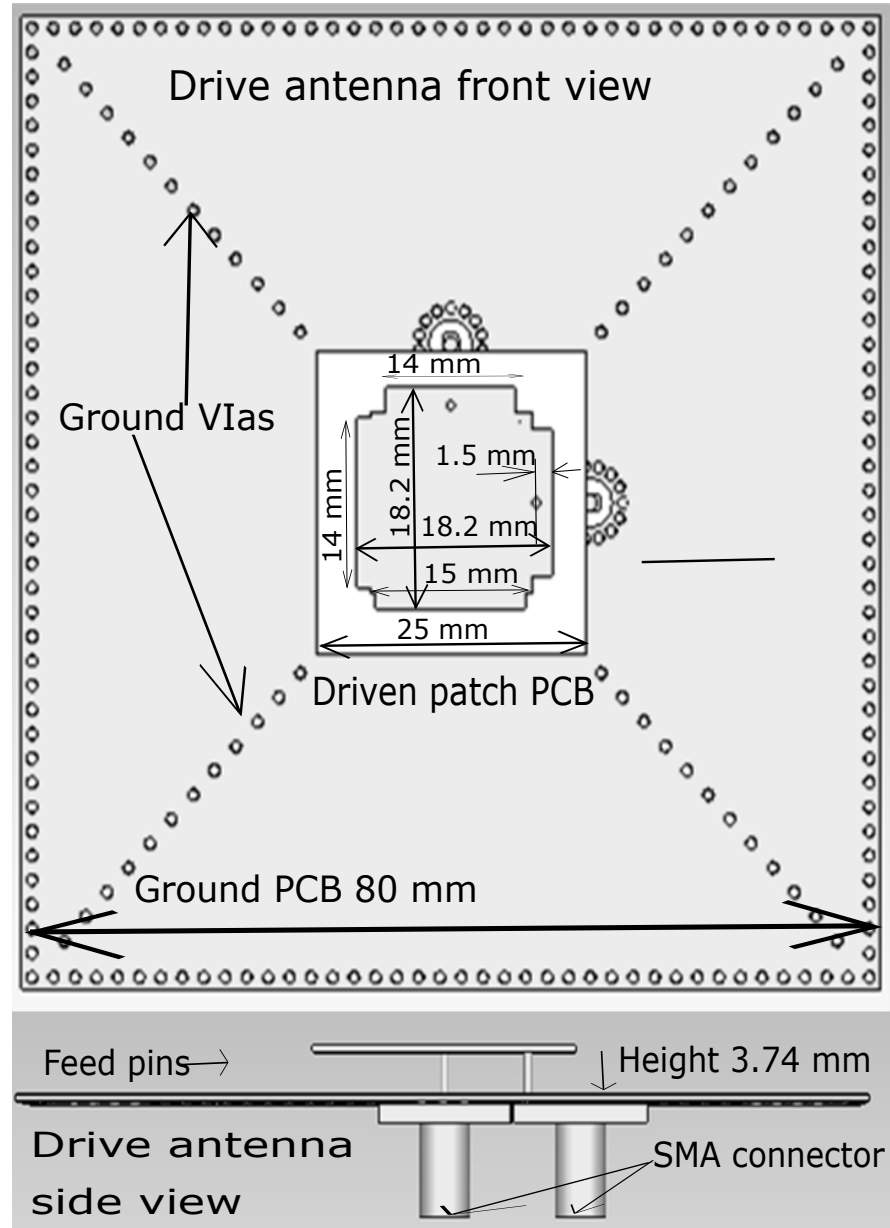

Figure 1. Drive patch, feed structure and ground PCB.

other end of the transmission line is galvanic contact to the SMA connector center pin. This $50 \mathrm{ohm}$ transmission line is $6 \mathrm{~mm}$ long. Antenna feeding points for two orthogonal polarization are transferred in a distance to accommodate two SMA connector at the bottom of the ground PCB. This extra transmission line introduce a small loss from coaxial cable feed point to antenna excitation point. This will help to attach one $50 \mathrm{ohm}$ load to one connector while measuring other antenna port. SMA connectors were placed at the bottom of the ground PCB to have minimum effect during antenna radiation properties measurement and give reliable radiation patterns from the DUT. As the antenna is designed for $7 \mathrm{GHz}$ all dimensions on the $\mathrm{PCB}$ have significant role. CPW feed line is chosen to reduce radiation from transmission line. This minimize loss and maintain polarization purity. To keep proper distance between patches, supporting parts are made of lowdielectric and low-loss Rohacell $31 \mathrm{HF}$ material. Measured dielectric properties of this rohacell at $10 \mathrm{GHz}$ are $\varepsilon_{r}=1.04$ and $\tan \delta=0.0017$. Transition between CPW line and SMA connector is done by ground opening and via implementation around SMA connector model. Fig. 2 also shows manufactured antenna array. A small plastic base is glued at the bottom of the ground PCB to give the PCB and SMA rigidness as PCB substrate thickness is only $0.508 \mathrm{~mm}$. Antenna feed pins are 
soldered to CPW line and drive patch. Small slots were cut in the dielectric support towers to place the vertical patches. A top dielectric cover is used at the top to keep all towers together in right spacing. Bottom parts of dielectric towers are glued to ground PCB.

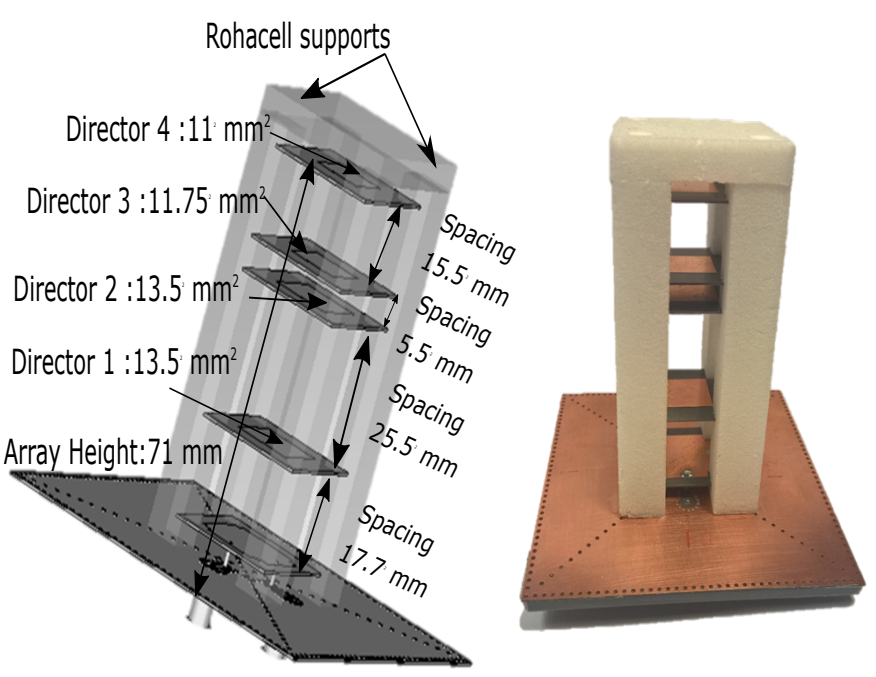

Figure 2. Stack patch antenna array simulation model and manufactured proto.

\section{Non-uniform spacing between directors}

In the proposed four stacked director patch antenna array design, the director's spacing is non-uniform. Proposed array directors are chosen by a parametric (patch size and gap) optimization for gain and bandwidth enhancement. Each director has been checked by simulation to get better gain over the maximum bandwidth. Optimized 4 director spacing are shown in Fig. 2. Gain optimization by uniform spacing between directors is described in [16]. A comparative simulation results for gain over the bandwidth among directors spacing is shown in Fig. 3. In this comparative simulation drive and directors patches are the same as in proposed design only spacing between director patches are changed. It shows that proposed design spacing has higher gain over wider bandwidth.

\section{SIMULATION AND MEASURED RESULTS}

\section{A. Radiation mechanism}

Simulated E- field plots for port 1 and port 2 excitation at port vertical planes are shown in Fig. 4. We can observe that strongest electric field is in drive patch. 1st, 3rd and 4th directors have stronger field compare to 2nd director. From this near field plot we can see how field directivity increases with number of directors in vertical direction.

\section{B. S-parameter and maximum Gain}

The S-parameters of the prototype are measured by a vector network analyzer from the two antenna ports. Measured and simulated S-parameter are shown in Fig. 5 and they agree

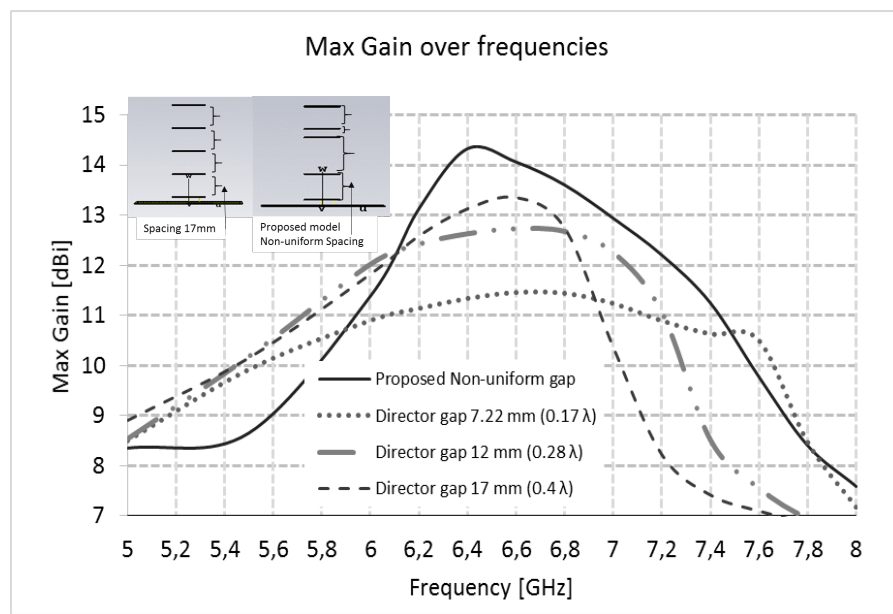

Figure 3. Simulated gain plots over frequencies for different director spacing.

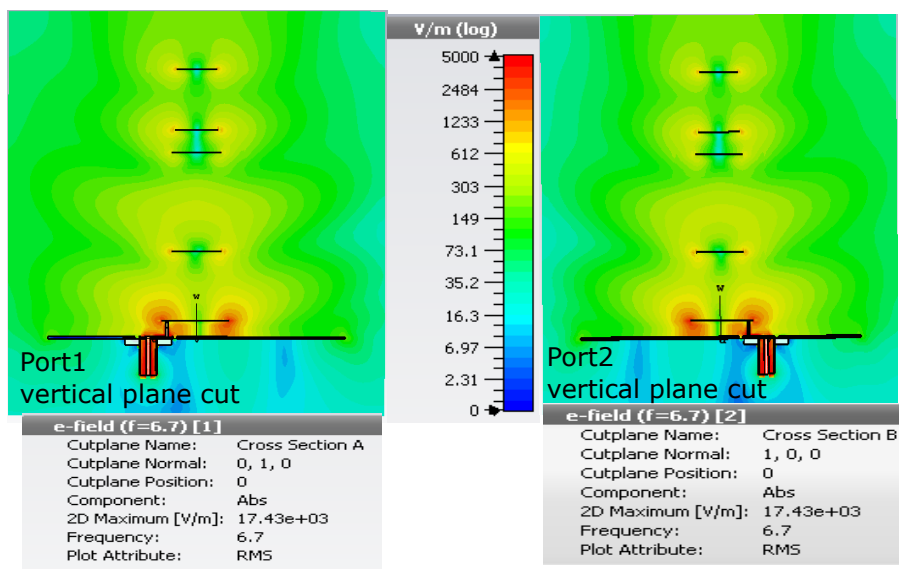

Figure 4. Simulated electric field across the cut planes for two ports.

well. Measured impedance matching at the band of interest is reasonable. Isolation between the ports is above $10 \mathrm{~dB}$ at the band of interest. Antenna radiation properties have been measured by the (Satimo StarLab) near field measurement system. Measured realized max gain is also plotted in Fig. 5. Both ports maximum gain reach more than $12 \mathrm{dBi}$. Small deviation of gain at port 2 is observed. This might come from manufacturing inaccuracy at port 2 . There is a frequency offset between maximum gain of the antenna and the best impedance matched frequency. Considering the loss of small transmission lines, connector and the miss-match loss good agreement of measurement and simulation is observed.

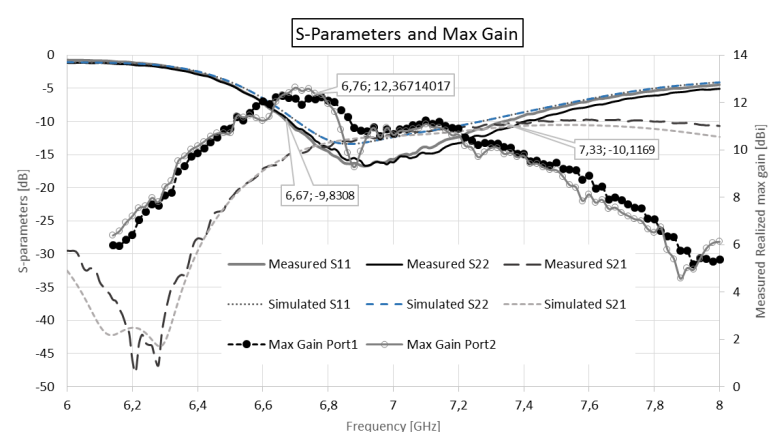

Figure 5. Simulated and measured S-parameters and measured realized maximum gain. 


\section{Antenna total efficiency}

Simulated and measured total efficiency is shown in Fig. 6. From both simulation and measured results it is observed that total efficiency correspond to input impedance matching and has same performance as in the simulation over the frequencies. This antenna has more than $90 \%(-0.4 \mathrm{~dB})$ efficiency at some frequency points.

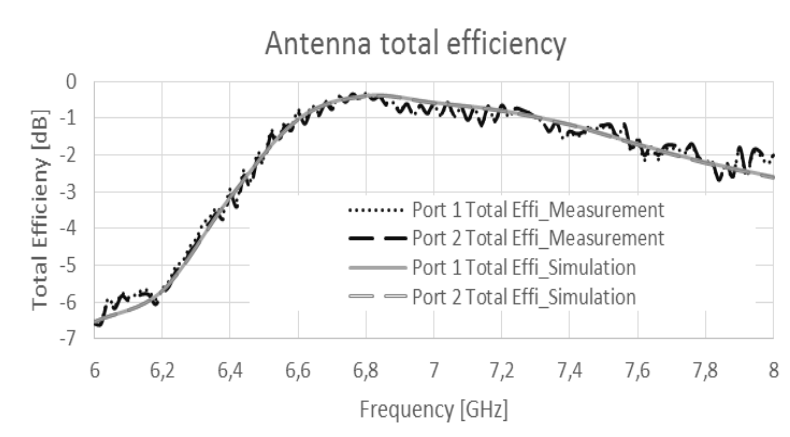

Figure 6. simulated and measured total efficiency.

\section{Radiation patterns}

Measured radiation patterns at major plane cuts for two ports at $6.7 \mathrm{GHz}$ are shown in Fig. 7. Good front to back ratio and stable symmetric radiation patterns in two plane cuts are observed. Toward the main beam co- and cross-polarized components have more than 12 difference for both ports.

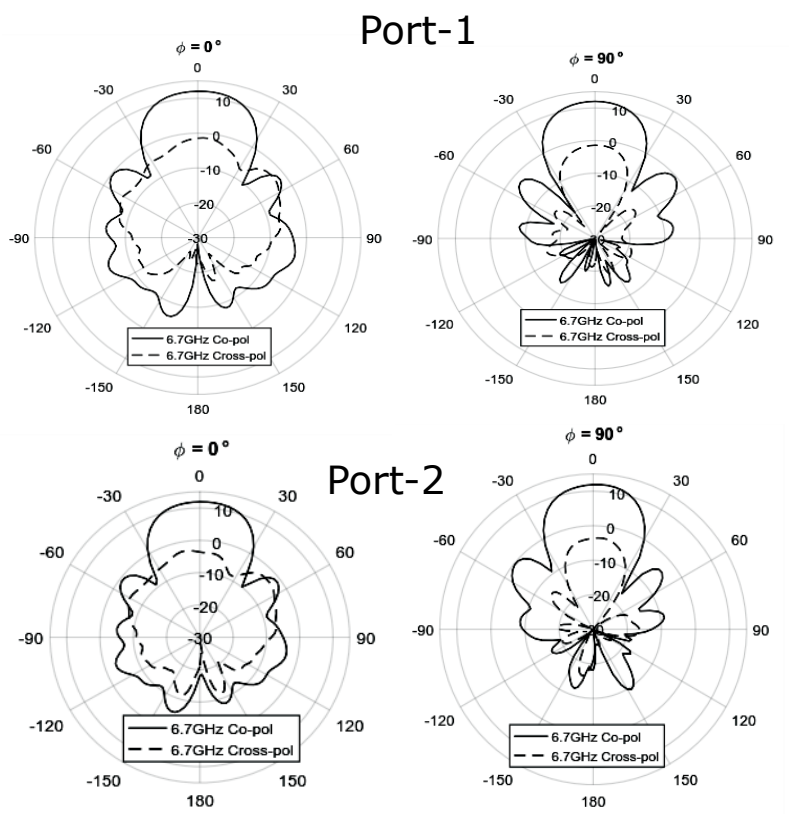

Figure 7. Measured radiation patterns cuts at $6.7 \mathrm{GHz}$.

\section{ISOLATION IMPROVEMENT PROPOSAL}

\section{A. Drive patch design}

A unique drive patch with L-probe fed is found which can be used to improve isolation between two ports. Stack patch array with this drive patch is shown in Fig. 8. Drive patch detail dimensions are shown in Fig. 9. This drive patch is simulated with $0.18 \mathrm{~mm}$ thick FR4 substrate and excitation ports are $100 \mathrm{Ohm}$. In this stack patch array all others directors patch shape and distance are kept same as reported measured antenna in Fig. 2. With this simulated antenna structure we showed that this measured antenna isolation can be improved by changing drive patch and feeding method.

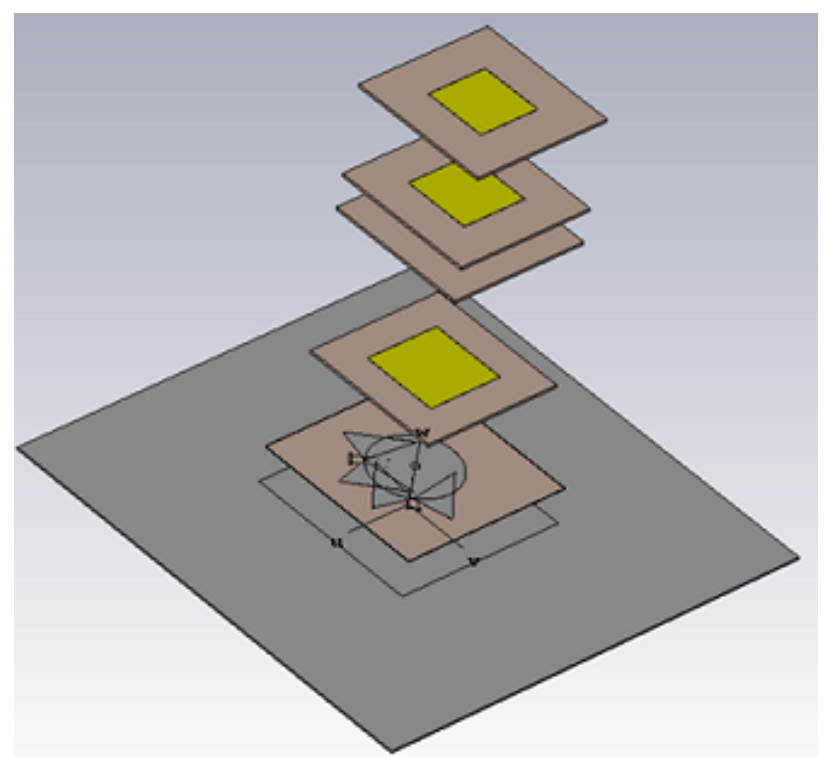

Figure 8. Stack patch array with isolation improved drive patch.
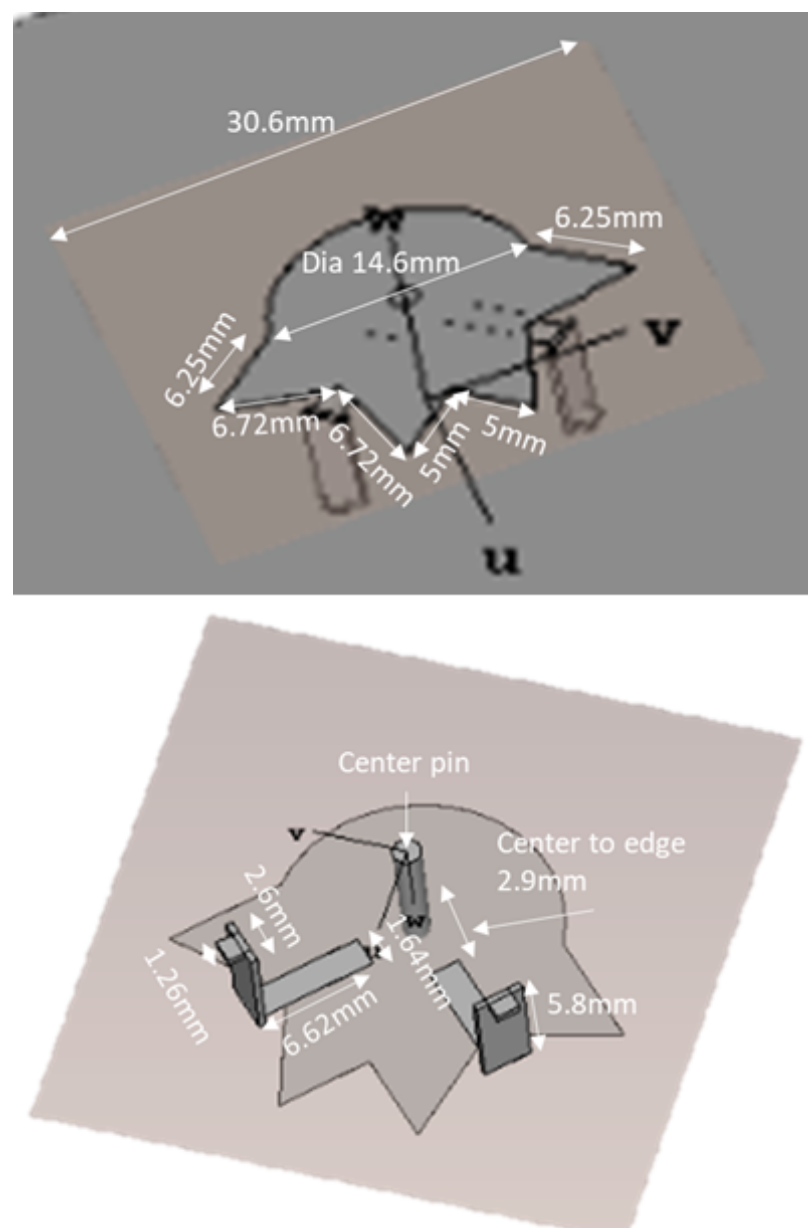

Figure 9. Simulated drive patch detail dimension with $\mathrm{L}$ probe fed. 


\section{B. S-parameter of simulated antenna}

The S-parameters of the simulated array is shown in Fig. 10. Both ports have $-10 \mathrm{~dB}$ matching for $1 \mathrm{GHz}$ bandwidth and both have the same value because of symmetrical structure from both feeds. In this model we can see that isolation is more than $15 \mathrm{~dB}$ between ports. This simulated drive patch shows a solution to improve the isolation compare to measured antenna. Antenna radiation properties like maximum gain over the frequency have been calculated in the simulation model. Calculated maximum gain over the frequencies for both feeds is plotted in Fig. 11. Both ports maximum gain reach more than $13 \mathrm{dBi}$ in this simulation model. This result also shows that this has similar gain over the bandwidth as in measured antenna proto. Radiation patterns for both ports are similar as in Fig. 7.

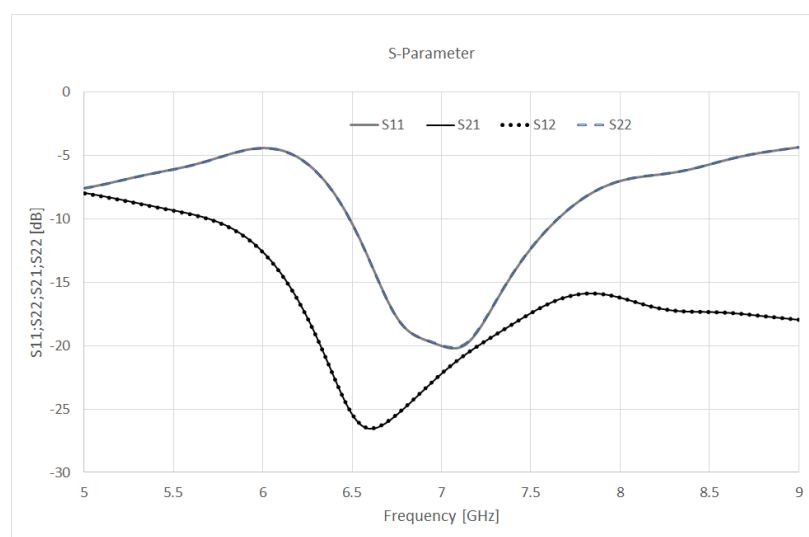

Figure 10. Simulated S-parameter.

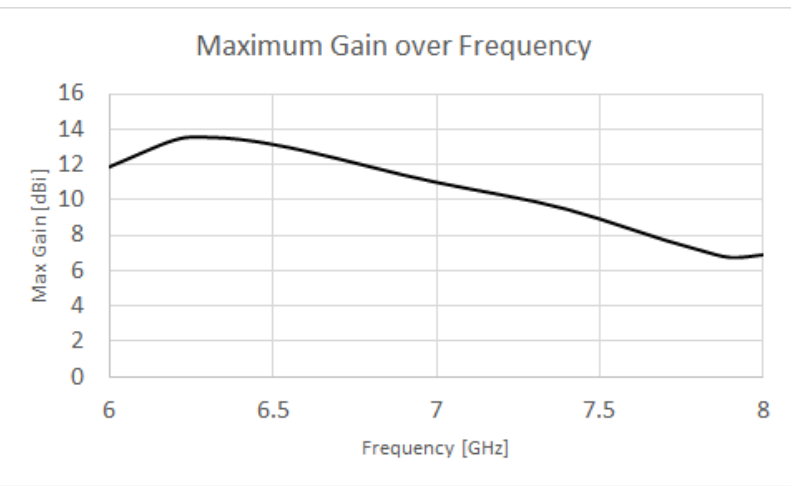

Figure 11. Simulated Maximum Gain over Frequencies.

\section{CONClusion}

A unique vertically asymmetric spacing stacked patch antenna is demonstrated with its optimization technique. Such high gain and dual polarized simple Yagi-Uda stacked patch antenna design has not found in any literature. This design approach can be very useful for high gain requirement wireless antenna solution such as $5 \mathrm{G}$ antenna deployment at $28 \mathrm{GHz}$ frequency range. Improvement of the isolation between the ports is proposed by a simulated drive patch antenna model.

\section{REFERENCES}

[1] G. Thiele, "Analysis of yagi-uda-type antennas," IEEE Transactions on Antennas and Propagation, vol. 17, no. 1, pp. 24-31, 1969.
[2] E. A. Jones and W. T. Joines, "Design of yagi-uda antennas using genetic algorithms," IEEE Transactions on Antennas and Propagation, vol. 45, no. 9, pp. 1386-1392, 1997.

[3] D. Cheng and C. Chen, "Optimum element spacings for yagi-uda arrays," IEEE Transactions on Antennas and Propagation, vol. 21, no. 5, pp. 615-623, 1973.

[4] C. Chen and D. Cheng, "Optimum element lengths for yagi-uda arrays," IEEE Transactions on Antennas and Propagation, vol. 23, no. 1, pp. 8$15,1975$.

[5] A. P. Gorbachev, O. O. Kibirev, and V. S. Churkin, "A modified broadband planar quasi-yagi antenna," in Actual Problems of Electronic Instrument Engineering (APEIE), 2010 10th International ScientificTechnical Conference on. IEEE, 2010, pp. 46-48.

[6] D. Arceo and C. A. Balanis, "A compact yagi-uda antenna with enhanced bandwidth," IEEE Antennas and Wireless Propagation Letters, vol. 10, pp. 442-445, 2011.

[7] J. Yeo and J.-I. Lee, "Bandwidth enhancement of double-dipole quasiyagi antenna using stepped slotline structure," IEEE Antennas and Wireless Propagation Letters, vol. 15, pp. 694-697, 2016.

[8] M. N. Islam, M. Berg, T. Tarvainen, and E. T. Salonen, "Wide band 1-probe fed circular patch antenna with elliptical parasitic patch and two elements array," Progress In Electromagnetics Research C, vol. 60, pp. 169-177, 2015.

[9] R. Q. Lee and K.-F. Lee, "Experimental study of the two-layer electromagnetically coupled rectangular patch antenna," IEEE Transactions on Antennas and Propagation, vol. 38, no. 8, pp. 1298-1302, 1990.

[10] J. Huang and A. C. Densmore, "Microstrip yagi array antenna for mobile satellite vehicle application," IEEE Transactions on Antennas and Propagation, vol. 39, no. 7, pp. 1024-1030, 1991.

[11] G. R. DeJean and M. M. Tentzeris, "A new high-gain microstrip yagi array antenna with a high front-to-back (f/b) ratio for wlan and millimeterwave applications," IEEE Transactions on Antennas and Propagation, vol. 55, no. 2, pp. 298-304, 2007.

[12] Z. Yang, K. C. Browning, and K. F. Warnick, "High-efficiency stacked shorted annular patch antenna feed for ku-band satellite communications," IEEE Transactions on Antennas and Propagation, vol. 64, no. 6 , pp. 2568-2572, 2016.

[13] Y.-X. Guo, K.-M. Luk, and K.-F. Lee, "L-probe fed thick-substrate patch antenna mounted on a finite ground plane," IEEE Transactions on Antennas and Propagation, vol. 51, no. 8, pp. 1955-1963, 2003.

[14] L. Ge and K. M. Luk, "A wideband magneto-electric dipole antenna," IEEE Transactions on Antennas and Propagation, vol. 60, no. 11, pp. 4987-4991, 2012.

[15] Y. Liu, H. Liu, M. Wei, and S. Gong, "A novel slot yagi-like multilayered antenna with high gain and large bandwidth," IEEE Antennas and Wireless Propagation Letters, vol. 13, pp. 790-793, 2014.

[16] O. Kramer, T. Djerafi, and K. Wu, "Vertically multilayer-stacked yagi antenna with single and dual polarizations," IEEE Transactions on Antennas and Propagation, vol. 58, no. 4, pp. 1022-1030, 2010. 